\title{
Moyamoya disease with neuro-ophthalmic manifestations: a rare case report
}

This article was published in the following Dove Press journal:

Eye and Brain

15 May 2010

Number of times this article has been viewed

\author{
Dipankar Das' \\ Sanjeev Kumar Handique ${ }^{2}$ \\ Harsha Bhattacharjeel \\ Sanjoy Kumar Buragohain' \\ Gayatri Bharali' \\ Akshay Chandra Deka' \\ Sunil Kumar Singh' \\ 'Sri Sankaradeva Nethralaya, \\ Guwahati, Assam, India; ${ }^{2}$ Department \\ of Radiology and Imaging, Guwahati \\ Neurological Research Centre and \\ Hospital, Assam, India
}

Correspondence: Dipankar Das Department of Ocular Pathology, Uveitis and Neuro-Ophthalmology Services Sri Sankaradeva Nethralaya, Beltola, Guwahati - 781028, Assam, India

$\mathrm{Tel}+9|036| 2228879$

$\mathrm{Fax}+9|036| 2228878$

Email dr_dasdipankar@yahoo.com

\begin{abstract}
Moyamoya (meaning a hazy puff of smoke) disease (MMD) is a rare, idiopathic, persistent, occlusive cerebrovascular disease involving bilateral progressive stenosis or occlusion of a terminal portion of the internal carotid artery, or a proximal portion of the anterior cerebral arteries and the middle cerebral arteries. There are irregular perforating vascular networks (moyamoya vessels), seen in the base of the brain, which produce magnetic resonance images of this 'puff of smoke' condition. Although MMD is prevalent mostly in Japan, it is also occasionally seen outside Asia. We report an interesting case of bilateral optic nerve involvement of a radiologically diagnosed case of MMD in a young lady seen in the north-east part of India.
\end{abstract}

Keywords: magnetic resonance angiography, stroke, optic atrophy

\section{Introduction}

Moyamoya disease (MMD) is an uncommon occlusive cerebrovascular disorder characterized by progressive stenosis or occlusion of the distal internal carotid artery (ICA) and proximal cerebral arteries, with a widespread net-like arrangement of cerebral collaterals. Takeuchi and Shimizu first described the entity in $1957^{1}$ and Suzuki and Takaku named the disease after Japanese term "moyamoya" in 1969. ${ }^{2}$ The appearance of an abnormal fine vascular network in catheter angiography was described as 'a hazy cloud like a puff of cigarette smoke'.3,16 MMD is an idiopathic disorder and has to be differentiated from angiographic correlates of other entities such as arteriosclerosis, autoimmune disease, trisomy 21, phakomatoses, head trauma, brain neoplasm, sickle cell disease etc. The incidence of MMD is reported to be 0.94 patients per 100,000 Japanese populations, with a prevalence of 10.5 patients per $100,000 .^{3,4}$ A recent survey showed women are more commonly affected by MMD, with a female to male ratio of 1.8:1. ${ }^{4}$ The onset of MMD is bimodal in age distribution with first peaks in childhood and second peaks affecting adults in their fourth decade of life. ${ }^{5}$ The Ministry of Health and Welfare of Japan has defined four types of MMD: ischemic, hemorrhagic, epileptic and "other". $6,7,14$ A predilection for ischemic cerebrovascular events is seen in childhood, and hemorrhagic strokes are common in adults. ${ }^{6,7}$

We report a rare case of MMD with bilateral optic nerve involvement in a young lady from the north eastern part of India.

\section{Case report}

A 25-year-old lady came to the neurophthalmology clinic of a referral institute in northeast India with chief complaints of headache and sudden painless dimness of vision of the 
right eye (OD) for the previous six days. She had experienced frequent transient loss of vision for about 15-30 seconds, on average 2-3 times per day for the previous week, before sudden loss of vision of that eye. Her left eye (OS) vision had been poor for the previous four years. There was no history of head injury, seizures, unconsciousness, jaw claudication, scalp tenderness, muscle aches, joint pain, anorexia, weight loss, hypertension, diabetes, skin changes, weakness, or fever.

On ocular examination, best-corrected visual acuity (BCVA) was finger counting (FC) 2 feet, $\mathrm{N}<36$ in $\mathrm{OD}$ and FC 2 meters, N.24 in OS. Ocular alignments and extraocular movements were normal with grade 1 relative afferent pupillary defect (RAPD) noted in OS. Slit lamp examination revealed normal anterior segments in both eyes with normal intraocular pressure in applanation tonometry. Vitreous cavity was unremarkable in both eyes. Dilated fundus examination of both eyes showed optic disc edema in OD and mild optic nerve pallor in OS (Figure 1). There was no associated peripapillary or retinal hemorrhages.

Systemic evaluation by an in-house physician revealed normal higher functions and other normal systemic parameters. Previous laboratory investigations carried out elsewhere showed normal blood count, platelet, erthrocyte sedimentation rate (ESR), C-reactive protein, peripheral blood smears, antinuclear antibody (ANA), lipid profile and random blood sugar. Veneral disease research laboratory (VDRL) was nonreactive. The patient was given magnetic resonance imaging (MRI) and visual-evoked potential (VEP). MRI revealed multiple stenotic and occlusive lesions in the intracranial arteries with formation of multiple collaterals suggestive of moyamoya disease (Figure 2). There was a possible old infarct in the right frontal deep white mater. There was atrophy of the left optic nerve and chiasm probably due to chronic ischemic changes. VEP showed prolonged L1 latency in OS. Ishihara's color vision test was subnormal and showed gross R-G defect in both eyes. The patient was referred to a local neurological institute where a thorough neurological evaluation was

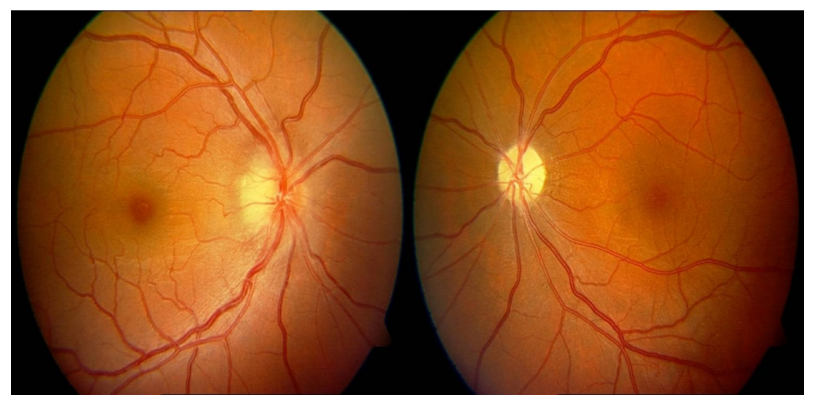

Figure I Fundus picture of both eyes showed optic disc edema in the right eye and optic disc pallor in left eye.

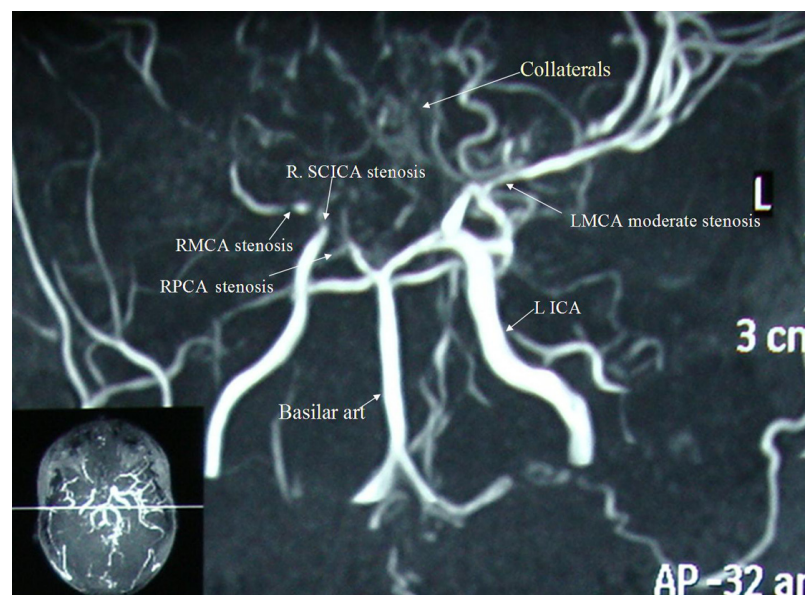

Figure 2 Magnetic resonance images (MRA and MRI) showing the features of MMD. The stenosis are seen in the figure.

Abbreviations: ICA, internal carotid artery; MCA, middle carebral artery; MRA, magnetic resonance angiograph; MRI, magnetic resonance imaging; SCICA, supraclinoid ICA.

performed. Currently, she is under close follow-up and has been prescribed oral aspirin $(150 \mathrm{mg})$ daily.

\section{Discussion}

MMD is seen in every continent and reported in almost all ethnic groups, but remains rare outside Japan. ${ }^{1,3,4,8,9}$ Our patient is a 25 year old housewife who presented with sudden painless dimness of vision in OD. She reported headache and recurrent episodes of amaurosis fugax in that eye prior to loss of vision. MMD is sometimes associated with migraine and headache is described as tension-type and these cases often require noninvasive MR examination to detect asymptomatic MMD.,11 Transient ischemic attack (TIA), cerebral infarction or intracranial bleeding can occur in both the pediatric and adult forms of MMD. ${ }^{9,10}$ Studies done in western countries showed that most patients were young woman unlike in Asia, where children were affected. ${ }^{10}$ Optic nerve involvements in MMD is a rare occurrence and it is mostly due to ischemia to the posterior ciliary arteries. Our patient had optic nerve edema in OD and optic atrophy in OS and this showed that the ischemic assault was progressing. There has been a single report of MMD with optic nerve pathology, also in a 51-year-old woman where the diagnosis of anterior ischemic optic neuropathy (AION) was made. ${ }^{4,15}$ The authors postulated that the most anterior part of the optic nerve head is supplied by the central retinal artery and its branches. Ischemia of this part leads to central retinal artery occlusion (CRAO) characterized by a pale retina with a cherry red spot in the macula. The prelaminar part was supplied by peripapillary short posterior arteries (SPCA) and peripapillary choriocapillaries. Ischemia of these arteries results in AION with optic nerve swelling resolving by 8 weeks which 
was followed by optic nerve pallor in sectoral distribution. In our case, we did not want to diagnose it as AION for a number of factors including the age of the patient.

The pathogenesis of MMD is still not clear. The general finding is of thickened intima of the major branches of the circle of Willis, and moyamoya vessels causing clinical symptoms. ${ }^{4}$ Ischemic events appear sometime after the onset of arterial occlusion or narrowing. Vascular endothelial growth factors (VEGF) expression is found to be inconclusive in collateral vascular network though VEGF has been related to other causes of cerebral angiogenesis. ${ }^{4}$

Asymptomatic MMD has been sporadically reported and there are no clear-cut guidelines on how to manage asymptomatic MMD. ${ }^{12}$ Studies had shown a possible genetic association of MMD linked to chromosome 17 in Japanese as well as in other demographics. Screening of family members of MMD had also identified small numbers of asymptomatic patients. ${ }^{12,13}$

Treatment of MMD depends upon its course. Cases with milder symptoms are usually treated conservatively. Severe symptomatic cases are generally treated using revascularization measures which include direct bypass, indirect and combined. ${ }^{4}$ Seventy-seven percent of cases are treated surgically because this has been shown to be more effective than nonsurgical treatment. ${ }^{4}$ Medical treatments include antiplatelet agents, vasodilators, antifibrolytic and antifibrinolytic agents. Epileptic episodes are treated by anticonvulsants. Ischemic episodes and thrombosis can be managed by antiplatelet agents, thus possibly preventing the progression of MMD. ${ }^{4}$ The present case was put on antiplatelet medication and further surgical options will be taken by the neurosurgeon as and when required.

MRI and magnetic resonance angiography (MRA) have been acknowledged as noninvasive diagnostic modalities for MMD. According to the guidelines for diagnosis of MMD established and modified by the Research Committee of the Ministry of Health and Welfare of Japan, conventional invasive cerebral angiography is not mandatory if MRI or MRI angiography clearly demonstrates all the findings. ${ }^{14}$ There are a total of six angiographic stages described in MMD. ${ }^{4}$ Our patient had stages 1 and 2 ie, there was bilateral stenosis or occlusion of internal carotid artery and abnormal vascular network at the base of the brain. Angiography is part of the standard diagnostic work-up

Eye and Brain

\section{Publish your work in this journal}

Eye and Brain is an international, peer-reviewed, open access journal focusing on clinical and experimental research in the field of neuro-ophthalmology. All aspects of patient care are addressed within the journal as well as basic research. Papers covering original research, basic science, clinical and epidemiological studies, reviews and evaluations, for evaluation of ischemic or hemorrhagic stroke and should be the practice pattern to diagnose this entity and iits follow-up. MMD with optic nerve involvement is a still fairly unrecognized cause. Ophthalmologists may encounter this condition and it requires urgent neurological/neurosurgical treatment.

\section{Disclosure}

The authors report no conflicts of interest in this work.

\section{References}

1. Takeuchi K, Shimizu K. Hypogenesis of bilateral internal carotid arteries. No To Shinkei. 1957;9:37-43.

2. Suzuki J, Takaku A. Cerebrovascular 'moyamoya' disease showing abnormal net-like vessels in base of brain. Arch Neurol. 1969;20: 288-299.

3. Bonduel M, Hepner M, Sciuccati G, Torres AF, Tenembaum S. Prothrombotic disorders in children with moyamoya syndrome. Stroke. 2001;32:1786-1792

4. Gorden M, Burke BA, Allen M, et al. Moyamoya disease: a summary. Neurosurg Focus. 2009;26:1-8.

5. Suzuki J, Kodama N. Moyamoya disease: a review. Stroke. 1983;14: 104-109.

6. Ikezaki K, Han DH, Kawano T, Kinukawa N, Fukui M. A clinical comparison of definite moyamoya disease between South Korea and Japan. Stroke. 1997;28:2513-2517.

7. Chiu D, Shedden P, Bratina P, Grotta JC. Clinical features of moyamoya disease in United States. Stroke. 1998;29:1347-1351.

8. Yoshimoto T, Houkin K, Takahashi A, Abe H. Angiogenic factors in Moyamoya disease. Stroke. 1996;27:2160-2165.

9. Houkin K, Abe H, Yoshimoto T, Takahashi A. Is 'unilateral' moyamoya disease different from moyamoya disease? J Neurosurg. 1996;85:772-776.

10. Tagawa R, Naritomi H, Mimaki T, Yabuuchi H, Sawada T. Regional cerebral blood flow, clinical manifestations, and age in children with moyamoya disease. Stroke. 1987;18:906-910.

11. Park-Matsumoto YC, Tazawa T, Shimizu J. Migraine with aura-like headache associated with moyamoya disease. Acta Neurol Scan. 1999;100:119-121.

12. Nanba R, Kuroda S, Takeda M, et al. Clinical features and outcomes of 10 symptomatic adult patients with moyamoya disease. No Shinkei Geka. 2003;31:1291-1295.

13. Yamauchi T, Houkin K, Tada M, Abe H. Familial occurrence of moyamoya disease. Clin Neurol Neurosurg. 1997;99 suppl 2:S162-S167.

14. Fukui M. Guidelines for diagnosis and treatment of spontaneous occlusion of the circle of Willis (moyamoya disease). Research committee on Spontaneous Occlusion of the Circle of Willis (Moyamoya Disease) of Ministry of Health and Welfare, Japan. Clin Neurol Neurosurg. $1997 ; 2: 238-240$.

15. Chen CS, Lee AW, Kelman S, Wityk R. Anterior ischemic optic neuropathy in moyamoya disease: a first case report. Eur J Neurol. 2007; 14:823-825.

16. Kraemer M, Heienbook W, Berlit P. Moyamoya disease in Europeans. Stroke. 2008;39:3193-3200.

guidelines, expert opinion and commentary, case reports and extended reports are welcome. The manuscript management system is completely online and includes a very quick and fair peer-review system, which is all easy to use. Visit http://www.dovepress.com/testimonials.php to read real quotes from published authors. 